\title{
Development of a bioassay for continuous mon- itoring of TWIST1 activity
}

\author{
Jakub Czapiński1 ${ }^{1,2}$, Joanna Kałafut ${ }^{\bullet}$, Alicja Przybyszewska-Podstawka ${ }^{1}$, Marta Paw- \\ licka ${ }^{1}$, Anna Roszkowska ${ }^{1}$, Michał Kiełbus ${ }^{1}$, Lidia Borkiewicz ${ }^{1}$, Karolina Dudziak', \\ Arkadiusz Czerwonka ${ }^{1}$, Andrzej Stepulak ${ }^{1}$, Adolfo Rivero-Müller¹ \\ 1 Department of Biochemistry and Molecular Biology, Medical University of Lublin, Poland; \\ 2 Postgraduate School of Molecular Medicine, Warsaw, Poland; \\ * Correspondence a.rivero@umlub.pl; Tel.: +48-(81) 4486363 \\ - Equal contribution
}

Simple Summary: Epithelial-mesenchymal transition (EMT) is a process during which epithelial cells, characterized as immobile apico-basal polarized cells, acquire the motility of mesenchymal cells. EMT is a fluid process and cells can undergo the reverse process - mesenchymal-epithelial transition (MET). These are normal physiological processes that occur in early embryonic development, wound healing or placentation, but they can also be exploited by cancer cells during tumorigenesis, migration and invasion. One of the essential transcription factors that plays roles in EMT induction in various cancer cell types is TWIST1. Yet, there is a lack of bioassays to detect the bioactivity of this factor. The main aim of this work was to design and validate a system to assess TWIST1 activity in cells undergoing EMT/MET. The resulting system allows simple, inexpensive and real-time determination of TWIST1 activity in living cells as they undergo phenotypical transformation.

\begin{abstract}
TWIST1 is a transcription factor that affects cell behavior during development and cell differentiation. Yet, it is better known for its roles in neoplasia through regulation of cell plasticity. The pathological contributions of TWIST1 in tumor initiation, angiogenesis, invasion, metastasis, and chemo-resistance have been the focus of much research. To-date, the only way to quantitatively measure the abundance of TWIST is by immunoblots. Yet, no bioassay exists that can detect TWIST1 activity. Thus, we present here a TWIST1 cell-based assay that allows measuring the amount of active TWIST1 non-invasively in living cells. The bioassay was characterized against previously described TWIST1 "inhibitors", as well as by epigenetic modulators of TWIST1 gene expression. Moreover, we tested multiple cell lines, showing that the level of TWIST1 mRNA resembles that of the bioassay. We show that prostate cancer cells (PC3) undergoing EMT, migrate out of 3D-spheroids and have increased TWIST1 activity. This fast and reliable system to detect active TWIST1 in different biological conditions allows a detailed analysis of this factor, as well as it can be used for drug discovery, since TWIST1 is a potential target for cancer chemotherapeutics.
\end{abstract}

Keywords: TWIST1; reporter system; Gaussia Luciferase; EMT; cell-based assay 


\section{Cell-based TWIST1 Bioassay}

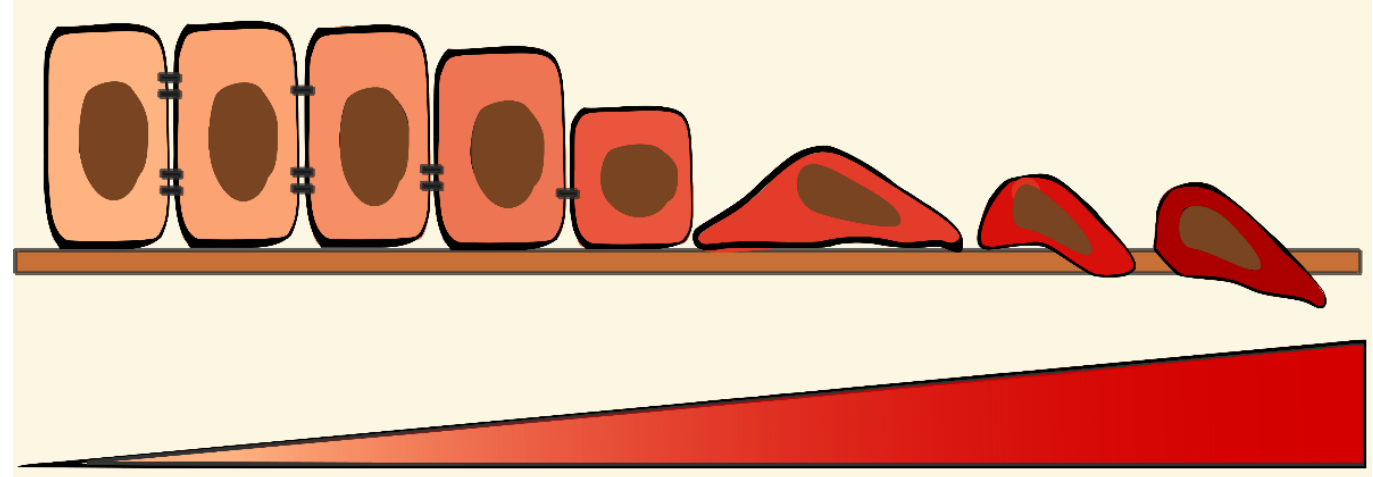

TWIST1 ACTIVITY

\section{Introduction}

TWIST transcription factors belong to the E-box binding proteins, a sub-group of the basic helix-loop-helix (bHLH) family, which also include the transcription factors SNAI1 and SLUG. Twist1 was first identified in Drosophila, where it acts as a crucial regulator for establishing dorsoventral polarity, mesoderm specification, and tissue-specific differentiation during early embryo development. The name was coined from the 'twisted' appearance of Drosophila embryos lacking Twist [1].

In humans, the most recognized role of TWIST1 is its participation in mesoderm-associated organogenesis - especially of limbs and craniofacial formation [1,2]. TWIST1 gene has two exons, and is located on the short arm of chromosome 7 at position 21.1. Mutations of the TWIST1 gene cause the Saethre-Chotzen syndrome (SCS), that is characterized by syndactyly, craniosynostosis, ptosis and hypertelorism [1,3]. Expression of TWIST1 has also been detected in stem cells within mesoderm-derived mesenchymal tissues (muscle, adipose tissue, and bone marrow) [1], or mesenchymal stem cells and in lymphocyte maturation [4]. In addition to the significant contribution of TWIST1 in normal developmental processes, this factor is also involved in multiple processes related to cancer development via activation of cell plasticity, particularly epithelial-to-mesenchymal transition (EMT).

EMT is currently the focus of intense research activities, due to the pronounced cell phenotypical changes in both physiological and pathological processes [1]. EMT accounts for the loss of apical-basal polarity as well as loss of cell-cell adhesion, promoting transition into an invasive, mesenchymal phenotype [1]. It is well established that TWIST1 contributes to the activation of EMT by promoting the expression of critical mesenchymal factors, such as N-cadherin, and by repressing epithelial ones such as E-cadherin [5-7]. This results in increased tumor cell motility and re-arrangements of the actin cytoskeleton, which coincides with cancer progression and metastasis [8,9]. Consistently, down-regulation of TWIST1 expression decreases invasion and migration of cancer cells[10]. Furthermore, TWIST1 has been under investigation for its tumorigenic properties and is highly considered to promote tumor development by inhibition of p53-dependent cell death, and 
therefore escaping senescence and apoptosis [11]. TWIST1 involvement in tumor angiogenesis and chemoresistance has also been reported [3].

TWIST1 expression is associated with the progression of several neoplasms of epithelial origin, such as breast cancer, particularly the HER2-positive subtype [4], ovarian [12], prostate [13-16] cervical [17], bladder [18],[19], hepatocellular [20], and colorectal cancer [21],[22]. It is specifically linked to head and neck cancers, including nasopharyngeal and esophageal squamous cell carcinoma [23-25],[26],[9]. Moreover, TWIST1 over-expression has also been reported in non-epithelial cancer types such as melanoma, glioblastoma, and chronic myelogenous leukemia (CML) [3],[27],[28],[29].

The role of TWIST as a prognostic marker for cancer progression and poor outcome has been explored in multiple studies. While TWIST1 overexpression correlates with poor outcome and decreased survival of patients suffering of ovarian, cervical, lung, oral and esophageal carcinomas [2],[30], its expression alone is not always in arrangement with either cancer progression or patients' poor prognosis, what has prevented using TWIST1 as a prognostic assessment marker for clinical applications such as patient stratification and therapy planning [2,3].

Although initial studies excluded TWIST1 as a drug target - mouse Twist1 knock-out is embryonic lethal and a mutation of one allele in humans causes SCS - later studies indicated the potential use of TWIST1 as a therapeutic target [31],[32],[33]. Currently, TWIST1 is pointed out as a target for the treatment of, among others, pulmonary sarcomatoid carcinomas and for alleviating muscle cachexia and related mortality in patients with advanced cancer. Nevertheless, there are no specific or selective inhibitors for TWIST1 available to-date. At best, a number of non-specific small molecules have been found to directly or indirectly inhibit TWIST activity [34],[35],[36],[37]. Here, we present and validate a bioassay for TWIST1 activity, developed with the goal to study the roles of TWIST during diverse biological processes. We show that TWIST1 activity is increased in cells migrating out of 3D organoids of prostate cancer cells, in line with previous reports $[13,38]$. This biosensor may also be a relevant cell-based assay for high throughput screening of smallmolecule inhibitors of TWIST, and their impact on cell plasticity.

\section{Materials and Methods}

Materials pSpCas9(BB)-2A-Puro (PX459) V2.0 was a gift from Feng Zhang (Addgene plasmid \# 62988 ; http://n2t.net/addgene:62988 ; RRID:Addgene_62988)[39]. The pmRmCherry vector was purchased from Clontech (CatNo 632542). The TGFB1-bio-His vector (proTGF $\beta$ ) was a gift from Gavin Wright (Addgene plasmid \# 52185 ; http://n2t.net/addgene:52185 ; RRID:Addgene_52185)[40]. E-cadherin-GFP was a gift from Jennifer Stow (Addgene plasmid \# 28009 ; http://n2t.net/addgene:28009 ; RRID:Addgene_28009)[41]. Flag Snail 6SA was a gift from Mien-Chie Hung (Addgene plasmid \# 16221 ; http://n2t.net/addgene:16221 ; RRID:Addgene_16221)[42]. KOD-Xtreme Hot-Start DNA polymerase was purchased from Merck Millipore. Restriction endonuclease DpnI was purchased from Thermo Fisher Scientific. Gibson Assembly Master Mix was purchased from New England Biolabs (NEB). The transfection reagent TurboFect was purchased from Thermo Fisher Scientific. All necessary antibiotics were purchased from Invitrogen. Cell culture media and supplements were purchased from Sigma. All PCR primers were bought from Genomed (Warsaw, Poland).

Molecular cloning All vectors were created using Gibson Assembly, according to the manufacture's protocol. In brief, PCR products were performed with a high-fidelity polymerase (KOD-Xtreme, Millipore), according to supplied protocol and then digested with DpnI FastDigest restriction enzyme (Thermo Scientific) to eliminate PCR templates (reduction colonies with original plasmids). Next, the PCR products were column purified 
(CleanUp and GelOut kit, Blirt) were mixed, at a ratio of insert/vector of 3/1, and added to the tube with $10 \mu \mathrm{l}$ Gibson Assembly Master Mix. After 15 minutes of incubation in $50^{\circ} \mathrm{C}$ electroporation of competent bacteria with $2 \mu \mathrm{l}$ of the reaction was performed. After 50 minutes incubation in $500 \mu \mathrm{l} \mathrm{SOC}$ medium (37॰C/100 RPM), bacteria were plated onto agar plates containing the recommended antibiotic (Ampicilin or Kanamycin). Dream Taq Dream Master Mix (Thermo Scientific) was used for colony screening PCR.

The TWIST1 binding domain (TWIST1BD) was synthesized as a DNA fragment (GeneArt Gene Synthesis) from Thermo Scientific.

The dCas9-VP64-T2A-Puro plasmid (CRISPRa) has been prepared in our lab. Gene encoding Cas9 has been mutated (D10A \& H840A) with the REPLACR method [43]. Next, downstream of dCas9, the VP64 gene has been cloned, using the Gibson Assembly method according to the manufacturer's protocol. The resulting DNA sequences were verified by sequencing.

The gRNAs sequences for CRISPR-Cas9 mediated gene knock-down were designed with Benchling, synthetized by Genomed (Warsaw, Poland) and then cloned into plasmids encoding the functional CRISPRa components, according to the Zhang's lab protocol [39], using T7 ligase and BbsI (both from Thermo Fisher).

CRISPRa The constructs used in the CRISPRa method is described above. For the experiment we designed two gRNAs, named gRNA1 (sequence: 5' - CACCGAATTGTTAGACCCCGAGGA-3') and gRNA2 (sequence: 5' - CACCGGACGAATTGTTAGACCCCG$\left.3^{\prime}\right)$ to target a genomic region around 100 nucleotides ahead of the start of transcription site for the TWIST1 gene. In the next step HEK-293T cells have been transfected and selected on puromycin for 14 days - until the stable cell line is settled. Then the cell line has been transfected with TWIST reporter system and after $48 \mathrm{~h}$ the media have been examined on GLuc activity.

Cell culture: Cell lines were cultured under standard conditions $\left(37^{\circ} \mathrm{C}, 5 \% \mathrm{CO}_{2}\right)$. The standard medium for culture of the HEK-293T cell line was DMEM (Sigma), while HEK293 and HeLa cell lines were cultured with medium EMEM (Corning), the HK-2 cell line was cultured with K-SFM medium (Invitrogen), and the RC-124 cell line with McCoy's 5a medium (Sigma). PC3 cells were cultured in DMEM-F12. All of the media were supplemented with 10\% Fetal Bovine Serum (Sigma) and 1\% Penicillin/Streptomycin solution (Sigma). In addition, McCoy's 5a medium was supplemented with $2 \mathrm{mM}$ L-glutamine. Transfections were carried out using the TurboFect reagent (Thermo Fisher). The ratio of DNA/transfection reagent was optimized for every cell line, in order to obtain the best transfection efficiency in the 24-well plate format.

TGF $\beta$ was produced in HEK293 cells, as previously reported [44], where the medium of cells transfected with a plasmid coding TGF $\beta$ was collected and transferred to 96 well plate containing cells expressing the TWIST1-GLUC reporter construct. As a control, we used media from mock-transfected HEK-293T cells. This eliminates the background resulting from the presence of other factors naturally secreted by this cell line.

Confocal imaging of living cells The cells were seeded onto 24-well glass-bottom plates (MoBiTec) one day before transfection. Transfection was conducted as described above. Transfected cells were then visualized under Nikon Ti Confocal microscope within $24 \mathrm{~h}$ after transfection using a $563 \mathrm{~nm}$ or $488 \mathrm{~nm}$ lasers.

RNA extraction, Reverse Transcription and qPCR Total RNAs was isolated using ExtractMe total RNA kit (Blirt) according to the manufacturer instructions. Next, cDNAs were synthesized using the High-Capacity cDNA Reverse Transcription Kit with addition 
of a RNase Inhibitor (Applied Biosystems) and used in qPCR. Glyceraldehyde 3-phosphate dehydrogenase (GAPDH) was used as a housekeeping gene that is stably and consistently expressed in most cell lines analyzed. Prior to use, all primer sets were tested for specificity and sensitivity, resulting in a single amplicon of the expected size. PCR reactions were then performed with the LightCycler® 480 II instrument (Roche) in triplicates on 96 well plates and PowerUp SYBR Green Master Mix (Applied Biosystems). The number of cycles needed to reach specific threshold of detection (CT) was used to calculate relative quantification (RQ). Relative mRNA expression was calculated using the delta CT subtraction and normalized to the expression of GAPDH.

Table 1. The primer sequences for qRT-PCR.

\begin{tabular}{lll}
\hline Gene & Primer sequence & \\
\hline GAPDH & $\begin{array}{l}\text { Forward } \\
\text { Reverse }\end{array}$ & 5'-CTCTGCTCCTCCTGTTCGAC-3' \\
& 5'-GCCCAATACGACCAAATCC-3' \\
TWIST1 & \\
& Forward & \\
& Reverse & $5^{\prime}$ - GCCGGAGACCTAGATGTCATT-3' \\
& & $5^{\prime}$ - TTTTAAAAGTGCGCCCCACG-3' \\
\end{tabular}

Gaussia Luciferase (GLuc) detection: The substrate for GLuc, coelenterazine, was purchased from Selleck, and dissolved in PBS to the final concentration $20 \mu \mathrm{M}$, incubated for 30 minutes/RT in the absence of light and used in a 1:1 ratio with cell culture media/supernatant. Detection of secreted GLuc was performed using a Tecan M200Pro with integration time $10000 \mathrm{~ms}$ and then analyzed. The results were normalized with flow cytometry analysis, MTT viability assay and $\beta$-Galactosidase Enzyme Assay System (Promega) according to the attached manual.

Chemical compounds: Tamoxifen and lactic acid were purchased from Sigma-Aldrich, Propofol 2\% MCT/LCT Fresenius from Fresenius Kabi.

Drug sensitivity assay (MTT viability assay): Yellow tetrazolium salt MTT (3-(4,5-dimethylthiazol-2-yl)-2,5-diphenyltetrazolium bromide) is metabolized by viable cells from yellow to purple formazan crystals. HeLa and HEK-293T cells for this test, were seeded on 96-well plates at a density of $2 \cdot 10^{4}$ (HeLa) and $4 \cdot 10^{4}(\mathrm{HEK}-293 \mathrm{~T})$ cells/ml in $100 \mu \mathrm{l}$ of a complete growth medium and cultured under standard conditions. After 24 hours, the culture medium was removed and the cells have been exposed to: tamoxifen $(2,4,6,8$ and $10 \mu \mathrm{M})$, propofol $(15,30$ and $60 \mu \mathrm{M})$, lactic acid (6 and $5 \mathrm{pH})$ and control without any additions, all in 8 replicates. All solutions have been prepared with supplemented growth media (DMEM F12, 10\% FBS, 1\% penicillin/ streptomycin). After 48 hours, the cells were incubated for 3 hours with a $5 \mathrm{mg} / \mathrm{ml}$ solution of MTT (Sigma). Formazan crystals were solubilized by adding 10\% SDS in $0.01 \mathrm{~N} \mathrm{HCl}$ and incubated for 3 hours on vortex. Detection the absorbance have been measured at a $570 \mathrm{~nm}$ wavelength using a Tecan M200Pro microplate reader.

Flow cytometry: The HEK293/T cells were seeded on 6-well microplates at a density of $5 \times 10^{5}$ cells $/ \mathrm{ml}$. After $24 \mathrm{~h}$ incubation, the cells have been exposed to tested drugs, and transfected by plasmids as described above. Next, the medium was removed and the cells were collected directly before the flow cytometric analysis (BD FACSCalibur). The AmCyan fluorescence intensity of individual cell was determined as SSC-H/FL2-H 2D- 
dot plots and used to transfection normalization. At least 10000 events were measured within an acquisition rate 100-300 events/second for each samples. The analyses were performed with the use of software CellQuest Pro Version 6.0. for the Macintosh operating system.

3D Spheroids Culture: PC3 cells 24h after transfection were seeded in a 96 Well Round (U) Bottom Plate, Sphera Low-Attachment Surface (Thermo Fisher) for a final concentration of 2,000 cells per well. Transfection was conducted as described above. Spheroids were grown within the human myoma-derived matrix (HMDM)/fibrin matrix containing the "Myogel" - a gift by Tuula Salo, Oulu University Hospital, University of Oulu and University of Helsinki [45]. The entire procedure of preparing the 3D cultures was conducted according to the protocol [46]. The cell migration was monitored for twelve days. Images were obtained using a Nikon Ti Confocal microscope. Each spheroid was visualized daily at the same microscope setting.

Statistical analyses: The data are expressed as means \pm standard deviation (SD). Statistical analyses were executed using GraphPad Prism 5.0 (GraphPad Software Inc.). The oneway analysis of the variance (ANOVA) and Tukey's Multiple Comparison Test were applied to the results of statistical comparison, where ${ }^{*} p$ values $<0.05,{ }^{* *} p$ values $<0.01$ and *** $\mathrm{p}$ values $<0.001$.

\section{Results}

Since TWIST1 is considered an essential factor for EMT, our goal was to generate a method that allows quantitative measurements of active TWIST1 in living cells without cell lysis. This is of great importance, since the EMT process is highly dynamic and multi-factorial. Thus, measuring changes in the expression or activity of EMT-promoting factors should ideally be monitored continuously throughout the process.

First, we designed an ideal DNA response element, to which the TWIST1 specifically binds in human promoters, using the PAZAR database [47]. Based on the specificity of binding, the regulatory region of the $Y B X 1$ gene was selected (Fig. 1A). This region (TWIST1 binding domain -TWIST1BD) contains three TWIST1 binding consensus sequences (CANNTG) (Fig. 1A). To test the effectiveness of this region, we placed it upstream of the gene coding for the mCherry fluorescent protein (Fig. 1B) as a reporter. Next, we carried out a series of transfections using several cell lines with a broad range of TWIST1 expression profiles. Subsequently, we compared the levels of TWIST1 mRNA versus the fluorescence intensity achieved by the mCherry reporter in every cell line.

A clear correlation between TWIST1 gene expression and the fluorescence level of mCherry in different transfected cell lines was found (Fig. 1E \& G). As changes of fluorescence intensity are more difficult to measure due to heterogeneity and linear progression, we decided to exchange $m$ Cherry for Gaussia Luciferase (GLuc), as this reporter enzyme is secreted by the cells into the medium, in which it can be readily detected and measured by luciferase assays without the need of cell lysis. This allows real-time, live cell and continuous cell-based assays that can be maintained and monitored for prolonged periods of time. Under these conditions, long-term assays can be performed that allow addressing phenotypic changes e.g. in response to inhibitors, changing cell culture conditions, or induced differentiation. Moreover, GLuc activity is higher than that of Firefly luciferase for about 1,000-fold [48]. The resulting vector - TWIST1BD-minPromoter-GLuc (Fig. 1C) was used for further analyses aimed at validating the system. As a starting point for further experiments, we performed identical analyzes as for the mCherry construct (Fig. 1E \& F). Additionally, we created a construct (minPromoter-GLuc) lacking the TWIST1 BD to visualize the effectiveness and specificity of the TWIST1 binding element (Fig. 1D). 
A.

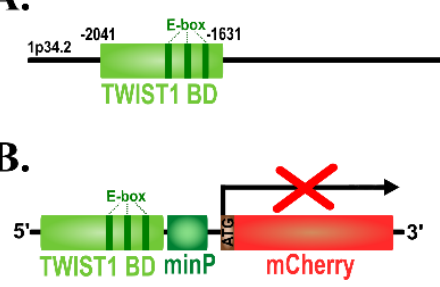

C.

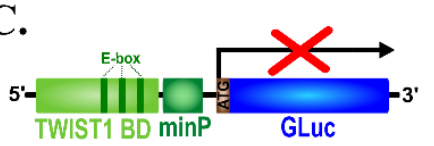

D.

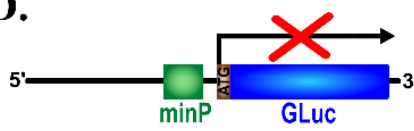

E.

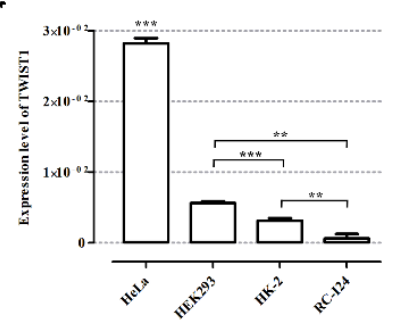

F.

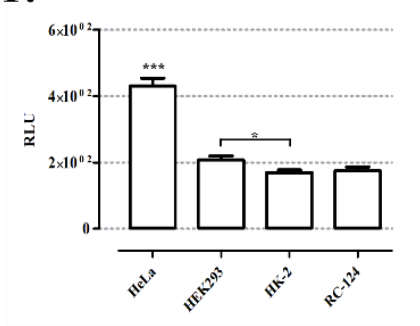

G.

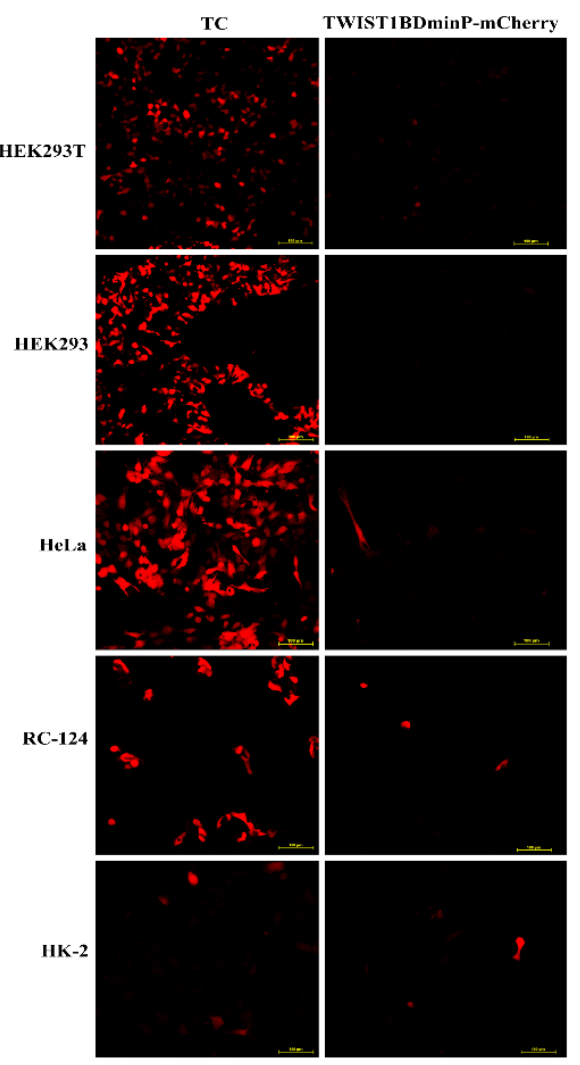

Figure 1. TWIST1 activity assay. A) Schematic representation of a YBX1 region. TWIST1 binding domain (TWIST1 BD) marked in green and E-boxes marked in dark green. B) Schematic representation of the action of a TWIST1-dependent mCherry reporter. C) Schematic representation of the inducible expression of a TWIST1-dependent reporter resulting in expression and subsequent secretion of Gaussia luciferase (GLuc). D) Schematic representation of the minimal promoter minPGLuc construct. E) qRT-PCR analysis of the TWIST1 mRNA levels in HeLa, HEK293, HK-2 and RC124 cells. The results show the mean $2^{-\Delta C T}$ of one run in triplicate \pm SD relative to GAPDH. F) Luminescence intensities, measured after $48 \mathrm{~h}$ of growth after transfection with TWIST1BD-minPromoterGLuc in various cell lines. At least 3 independent runs were performed for each experiment. The graph shows the representative result of the measurement. The results were analyzed with one-way ANOVA test and Tukey's Multiple Comparison post hoc test $\left({ }^{*} \mathrm{p}<0.05,{ }^{* *} \mathrm{p}<0.01\right.$, and ${ }^{* * *} \mathrm{p}<0.001$ were considered statistically significant). G) Expression of $m$ Cherry in cells transfected with TWIST1BD-minPromoter-mCherry (on right) and transfection control (TC) (on left).

As expected, the activity of GLuc in the media, as well as with mCherry fluorescence using the first assay architecture, followed a very similar pattern as that of TWIST1 mRNA expression - suggesting the sensor is able to quantitatively determine TWIST1 activity. As an internal control for transfection efficiency, we cloned a constitutively expressed nuclear localized fluorescent protein ( $3 x N L S-A m C y a n)$ under the CMV promoter ( $p C M V-3 \times N L S-$ AmCyan) cassette into the TWIST1-reporter plasmid.

TWIST1 activity is affected by multiple transcriptional and post-translational processes and crosstalk with other signaling molecules. At the transcriptional level there is several pathways involved in TWIST1 regulation among which TNF- $\alpha$, Wnt, TGF $\beta$, Notch, EGF or hypoxia are the best known examples [49],[50],[51]. The post-translational TWIST1 regulation is also tightly regulated by protein stabilization - phosphorylation (by MAPK, PBK, CK2) and degradation (by Ppa, FBLX14, p53-Pirh2 complex, PAQR3, Aurora kinase A) [8],[52]. Phosphorylation of TWIST1 has also its implications in dimer formation. TWIST1 may form homo- and heterodimers with proteins such as: E47, E12, TWIST2, Runx2, MyoD [53]. Thus, in order to determine whether our reporter system is responsive 
to TWIST1 activity and not just expression, we decided to use molecules that have been reported to modify the expression and/or activity of TWIST1 gene or protein. In the absence to any specific TWIST1 inhibitors, we chose four substances that have been reported to affect TWIST1 expression or activity: 1) lactic acid (reducing $\mathrm{pH}$ of cell culture medium) [54]; 2) TGF $\beta$ increases TWIST1 expression [55],[56], [57]; 3) Propofol, reduces the expression of TWIST1 mRNA [58]; and 4) tamoxifen, which accelerates the degradation of TWIST1 [59]. In addition to the pharmacological approach, we designed a molecular approach to directly activate the endogenous TWIST1 gene by using CRISPRa (CRISPR activation).

EMT is a complex process that can be activated or modulated by various external factors. One of such factors is lowering of the $\mathbf{p H}$, which is often observed in hypoxic cancer tissues. Inspired by the report by Tongwei Yang's group, we decided to use lactic acid to lower the $\mathrm{pH}$. Cells were transfected with TWIST1BD-minPromoter-GLuc. After, $24 \mathrm{~h}$ had elapsed, the culture media was exchanged by fresh culture media with reduced $\mathrm{pH}(6,0)$ or standard media (negative control - NC), and incubated for an additional period of 48 hours. Luciferase measurements show that by lowering the pH the TWIST1 activity increases (Fig. 2A). As lowering $\mathrm{pH}$ may affect cell viability, results have been normalized using a viability assay (Fig 2D).

The TGF $\beta$ pathway is one of the most effective inducers of EMT. As TWIST1 is a driving factor of EMT, we decided to test our reporter construct under TGF $\beta$-induced EMT. Induction of EMT by TGF $\beta$ in different lines expressing the TWIST1BD-minPromoter-GLuc reporter, showed a significant increase in luciferase signal (Fig. 2A).

Propofol does not affect the activity of the TWIST1 protein itself, but has been reported to downregulate expression of the TWIST1 gene what have been shown on the mRNA as well as protein level [58]. As expected, exposure to propofol resulted in a decreased expression of GLuc reporter; which was largely independent of the dose of the drug used in these experiments (Fig. 2B). Cells viability was unaffected by Propofol treatment (Fig. 2E).

Tamoxifen is the most widely selective estrogen receptor inhibitor used. Tamoxifen has also been reported to induce TWIST1 protein degradation, without changing its expression at the mRNA level [59]. In order to show the robustness and sensitivity of our reporter system not only for qualitative, but also for quantitative determination of TWIST1 activity, we decided to use six different concentrations of tamoxifen $(0,1,2,4,6,8$ and $10 \mu \mathrm{M})$ to reduce TWIST1 protein degradation. Samples were collected 24 and 48 hours after adding Tamoxifen to the cell culture. We observed a linear decrease in luminescence that correlates with increasing tamoxifen concentrations in the media (Fig 2C). These results have been normalized with respect to cell number and viability (Fig 2F).

There was no significant difference in luciferase activity in cells transfected with minPromoter-GLuc and treated with tamoxifen and propofol (Fig. $2 \mathrm{H}$ ), another indicator of the robustness and specificity of the reporter. 
A.

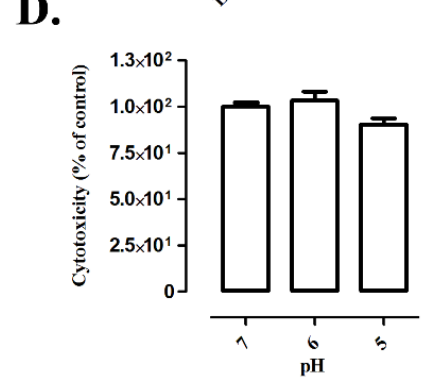

G.

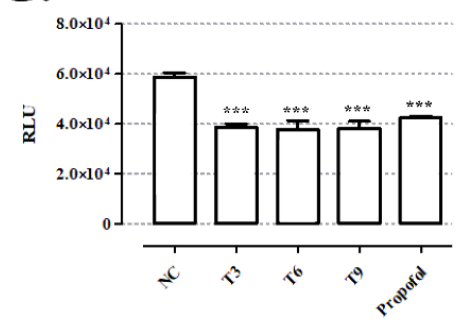

B.

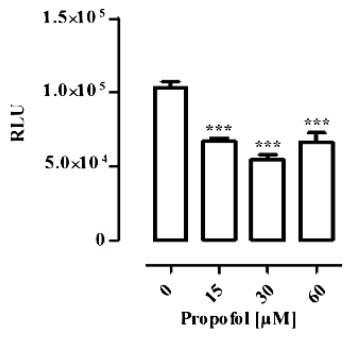

E.

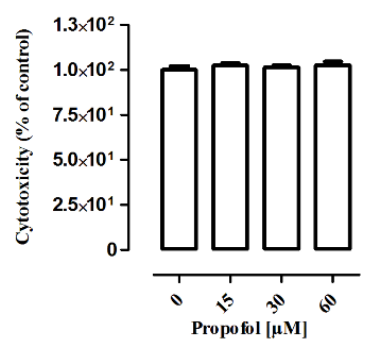

C.

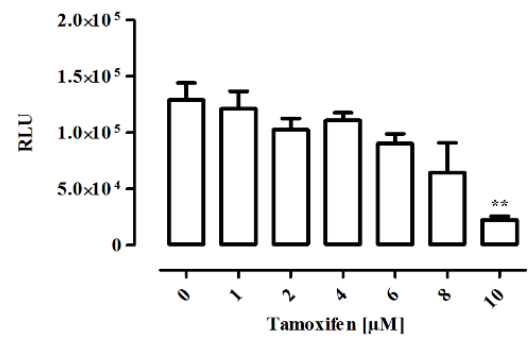

F.

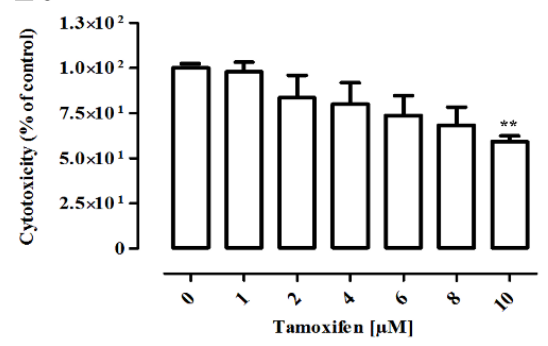

H.

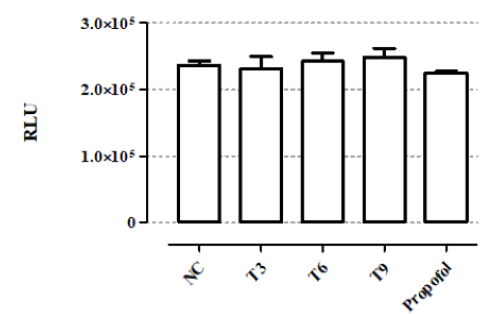

Figure 2. Pharmacological control of activity or expression of TWIST1 result in signal reduction in bioassay. A. Gaussia Luciferase activity in HEK293T after 48 hours of cell treatment by either lower $\mathrm{pH}(\mathrm{pH} 6$ by lactic acid) or TGF $\beta$, versus untreated cells (negative control, NC). B. GLuc activity from HeLa cells after 48 hours of treatment with propofol at multiple concentrations: 0 (control), 15, 30 and $60 \mu \mathrm{M}$. C. Tamoxifen at different concentrations: 0 (control), 1, 2, 4, 6, 8 and $10 \mu \mathrm{M}$. Viability is indicated for $\mathrm{pH} 6.0$ (D), Propofol (E) and tamoxifen (F) (MTT assay), in which untreated control cells were calculated as $100 \%$. G,H) Comparing the construct with full TWIST1BD (TWIST1BD-minPromoter-GLuc) with the construct without TWIST1BD (minPromoter-GLuc). GLuc activity in HEK293T transfected with TWIST1BD-minPromoter-GLuc (G) or minPromoter-GLuc (H) after 48 hours cell treatment with tamoxifen at different concentrations: 0 (negative control - NC), 3 (T3), 6 (T6), 9 (T9) $\mu \mathrm{M}$ and $15 \mu \mathrm{M}$ propofol. The results were normalized based on AmCyan expression using flow cytometry. The graph shows the representative result of the measurement. The results represent the mean $\pm \mathrm{SD}(\mathrm{n}=4)$ and were analyzed with one-way ANOVA test and Tukey's Multiple Comparison post hoc test $\left({ }^{*} \mathrm{p}<0.05,{ }^{* *} \mathrm{p}<0.01\right.$, and ${ }^{* * *} \mathrm{p}<0.001$ were considered statistically significant).

All of the tests descried above might also affect additional mechanisms and pathways, likely to indirectly affect TWIST1 expression and activity. Thus, we also used CRISPR activation (CRISPRa) for the final validation of our TWIST1 reporter system. CRISPRa is based on nuclease-dead Cas9 (dCas9), which retains its DNA-binding abilities, fused with transcriptional activator VP64 (Fig. 3A). The targeting genomic sequence is determined by the guide RNA (gRNA). HEK-293T cells stably expressing CRISPRa system components were transfected with the reporter plasmid and after 48 hours samples for GLuc activity were collected.

As presented in Fig. 3B, both $g R N A s$ induced the overexpression of TWIST1 although the second $g R N A$ ( $g R N A 2$ ) was $40 \%$ more efficient than $g R N A 1$. 
A.

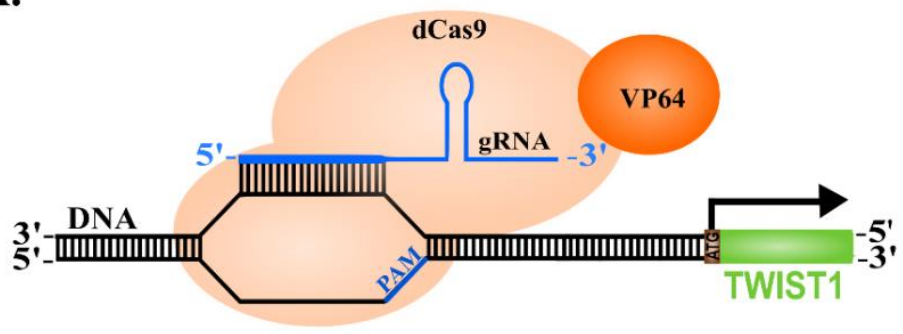

B.

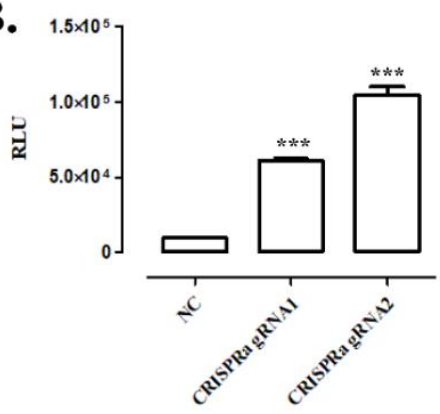

Figure 3. The design of the CRISPR/dCas9-VP64 activation (CRISPRa) system A. The CRISPRa system consists of a dead Cas9 (dCas9) protein, a specific guide RNA (gRNA), and the activation domain of the viral protein (VP64). B. CRISPRa targeting TWIST1 in HEK293T using two different gRNAs. The results represent the mean $\pm S D(n=4)$ and were analyzed with one-way ANOVA test and Tukey's Multiple Comparison post hoc test $\left({ }^{*} \mathrm{p} \leq 0.05,{ }^{* *} \mathrm{p} \leq 0.01\right.$, and ${ }^{* * *} \mathrm{p} \leq 0.001$ were considered statistically significant.

While construct TWISTBD-GLuc can be used to actually quantify TWIST1 activity, construct TWISTBD-mCherry may also be used to visualize the activity of this factor. Taking advantage of this, we assessed the activity of TWIST1 in organoid (3D) cultures, as it has been suggested that TWIST1 plays a role in EMT/migration of cells, we hypothesized that cells migrating out of the organoid will have a higher mCherry signal, due to higher TWIST activity, than those having a more epithelial phenotype within the spheroid. The cells were transfected with plasmids in 3 settings: to differentiate mesenchymal and epithelial cells - TWISTBD-mCherry + CDH1-GFP, to induce EMT - TWISTBD-mCherry + FlagSnail 6SA and a non-induced control TWISTBD-mCherry alone. To exclude errors in photo evaluation due to autofluorescence, we also used untransfected PC3 cells in 3D culture as a control (Figure S1). Cells after 24h were plated in 3D culture plates and observed for 12 days (see Materials and Methods section). As CDH1 is a marker strictly linked with epithelial state, it was expected that cells expressing CDH1, and with low TWISTBD-mCherry signal will be located inside spheroid (GFPHigh/mCherryLow cells ). In contrast cells undergoing EMT such as those overexpressing SNAIL will display increased motility and TWIST1 activity. The results shown in Figure 4 confirm our assumptions, the cells overexpressing CDH1-GFP are inside a spheroid and are clearly visible on the insets where green fluorescence do not overlap with red fluorescence of the reporter (even low TWIST1 activity). SNAIL-expressing cells on the next panel cells show high mobility and increased TWIST1 activity and reduced CDH1-GFP expression. Cells migrating outside the spheroid show high reporter red fluorescence and very low GFP (mCherry High/GFPLow). It is also clearly seen in the magnification (inset) that the leading cell highly expresses mCherry, showing its invasive state. 

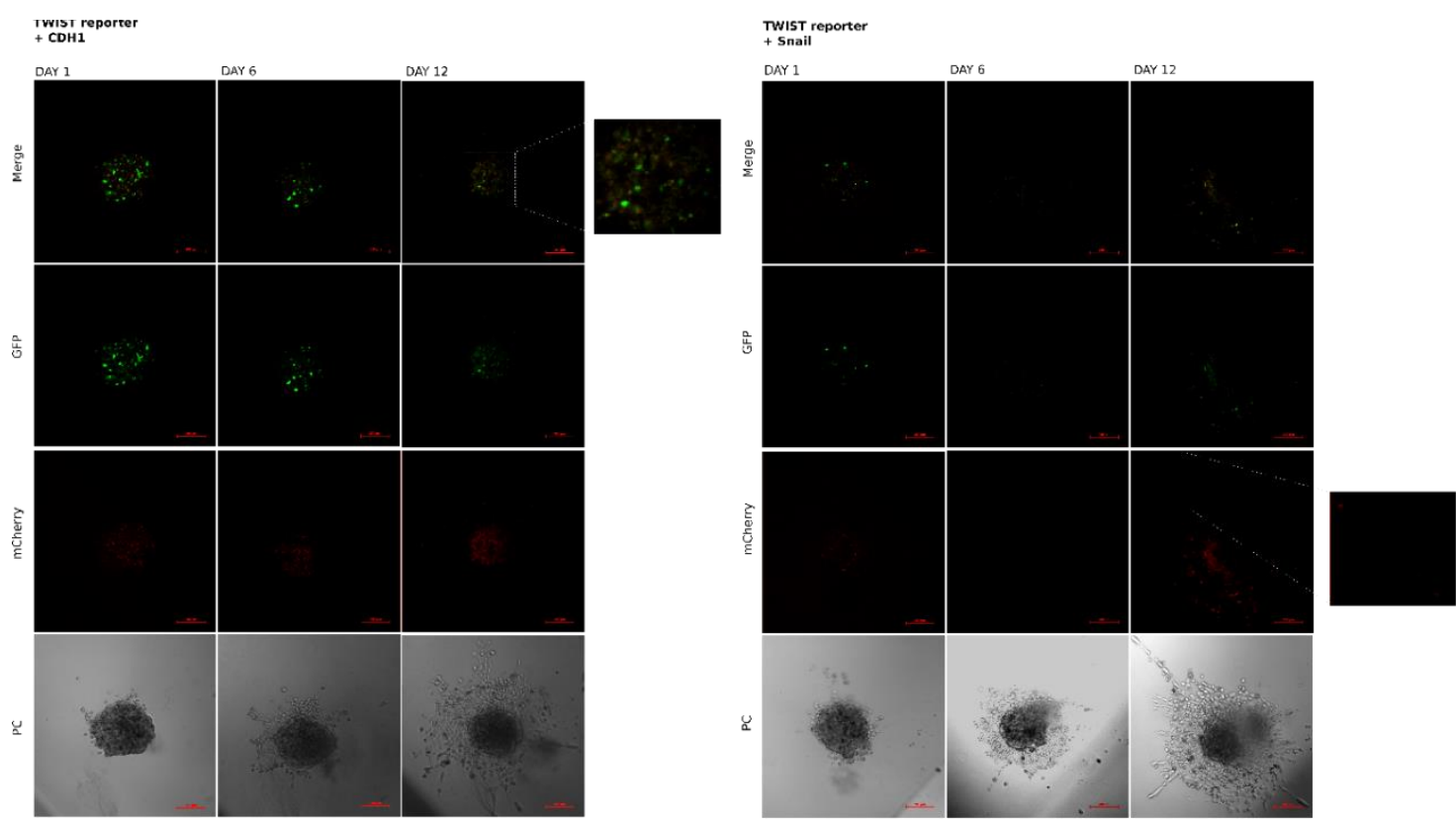

Figure 4. PC3 cells in 3D cultures. Cells were cotransfected with TWISTBD-mCherry/CDH1-GFP (left column) and TWISTBD-mCherry/CMV-3xNLS-AmCyan + Flag-SNAIL 6SA (right column). Spheroids were monitored for twelve days. The figure shows representative images of the same spheroid on day 1, 6 and 12 of culture. Green fluorescence localized in: nucleus indicates 3xNLS-AmCyan (both columns), whole cell - GFP (left column). Red fluorescence - mCherry (both columns). PC - phase contrast. Scale bar represents $200 \mu \mathrm{m}$.

\section{Discussion}

TWIST1 is an important transcriptional factor not only in the context of changes occurring during the initial developmental stages but also in many other processes such as angiogenesis, invasion, tumor initiation and progression, metastasis, acquisition of stemness, and acquired drug resistance $[1,8,49,60,61]$. To study the truly complex and multicontextual character of this versatile transcription factor, methods for visualization of its activity during cellular phenotypical changes are needed. Such tools would also be important to research its functional role in diverse context (e.g. in various tissues) and cell type-dependent manner. Multiple attempts to quantitatively measure the expression and activity of TWIST1 have been tested, for example by using TWIST1 promoter sequences followed by diverse reporter genes [62,63],[64]. Additionally, reporters containing TWIST1 3'UTR have been used for investigation of microRNAs affecting TWIST1 expression $[65,66]$. Yet, all these systems measure only TWIST1-promoter activity or mRNA stability and not TWIST1 activity. Moreover, assays based on firefly luciferase require terminal cell lysis, which is tedious, time-consuming and does not allow continuous monitoring of changes that take place in living cells. Such discontinuous methods are also not compatible with high throughput screening for drug discovery.

The cell-based TWIST1 bioassay presented in the current work, is composed by TWIST1 binding domain from YBX1 gene promoter, widely described TWIST1 target with the highest affinity score based on PAZAR database. As reporter systems we selected either a secreted Gaussia Luciferase, for continuous quantification, or a fluorescent protein for visualization purposes. For the latter, we incorporated $\mathrm{p} C M V-A m C y a n$ as an internal control of expression level and transfection efficiency.

The bioassays was validated versus gene expression in multiple cell lines and by synthetic transcriptional activation of TWIST1 by CRISPRa. Together with the use of two chemical "inhibitors" - Propofol and Tamoxifen, as well as inducers of EMT such as low $\mathrm{pH}$ and TGF $\beta$, we show that the biosensor is able to quantify TWIST1 activity in near real-time. 
Propofol is an anesthetic that shows a fairly complex mechanism of action. Currently, many scientific studies emphasize its action not only in the context of sedation but also in the impact on cancer treatment. The Yang group has presented evidence that propofol lowers TWIST1 at the mRNA and protein levels [58]. We thus tested whether propofol would reduce TWIST1 signaling, which it significantly did in all concentrations used. Taking into account that this compound does not work specifically on TWIST1, we decided to verify our system with another compound with an inhibitory effect of TWIST1 at the protein level, Tamoxifen, which is known to accelerate the degradation of TWIST1 through the ubiquitination-proteasome pathway without affecting transcription or translation. Our results are consistent with those by Jianming Xu and collaborators [59]. The activity of luciferase decreased in an inverse dose-dependent manner to the tamoxifen concentration.

We further tested how the induction of EMT affects TWIST1 activity. Here, once again, we selected two methods: 1) to lower $\mathrm{pH}$, which mimics pathophysiological conditions occurring inside solid tumors. Lower $\mathrm{pH}$ is a triggered of EMT and cell migration. In this experiment, the level of the system's luciferase has increased in terms of control. 2) The best described EMT activator- TGF $\beta$ - was used to trigger EMT. In both cases there was an increase of TWIST1 activity readout as compared to controls. Finally, to specifically increase TWIST1 expression, we decided to induce TWIST1 overexpression using CRISPRa. We designed two gRNAs, which guided dCas9-VP64 to the TWIST1 promoter area, providing the final proof for the selective operation of the system. All in all, our results are further supported by standardization with flow cytometry measurements, $\beta$-Galactosidase activity and viability assay, and are consistent with each other as well as previous reports.

Having demonstrated the advantages and possibilities of the Gaussia luciferase-based system, we decided to investigate possible applications of the mCherry-based system. To this end, we performed a series of transfections of prostate cancer cells (PC3) to induce overexpression of specific factor for the epithelial state (E-cadherin) and for the mesenchymal state (SNAIL) and culture them in conditions as close as possible to human environment using Myogel, a human-tumor derived origin matrix [45,67]. While cells overexpressing E-cadherin showed lower invasiveness and TWIST1 activity, SNAIL-expressing cells migrated out of the spheroid, as thus having a more mesenchymal phenotype, are these cells also expressed the TWIST reporter more actively, which, in line with previous research [68], providing more evidence that TWIST1 is an important player in mesenchymal transformation and migration.

Our bioassay shows robust correlation between TWIST1 gene expression and downstream protein activity. In addition, it shows that the reporter system can be applied for small molecule inhibitor screening, and that continuous real-time, live cell measurements at different time points are possible since the assay is not disruptive. Thanks to these features and the use of a secreted luciferase, the assay allows to reuse the cells for other analyses.

Since there are no specific TWIST inhibitors and the biology of TWIST1 is not completely known, visualization and/or quantification of its activity might help in the understanding of downstream processes in vitro and eventually in vivo.

Supplementary Materials: The following are available online at www.mdpi.com/xxx/s1, Figure S1: PC3 cell line 3D culture, Table S1: Primers sequences. RAW DATA file.

Author Contributions: Conceptualization, J.C., A.R.-M.,; Methodology, J.C., A.R.-M.; Validation, J.C., A.P-P., M.K., J.K., L.B., A.R., K.D., A.C.; Formal Analysis, J.C., J.K., A.P-P., A.C., M.P.; Investigation, J.C., J.K., A.P-P.; Resources, A.S., A.R.-M.; Writing-Original Draft Preparation, J.C., M.P., 
A.R.,; Writing-Review \& Editing, M.K., J.C., J.K., A.P-P. A.R.-M.; Visualization, J.K., M.K., J.C.; Supervision, A.R.-M., A.S.; Project Administration, A.R.-M.; Funding Acquisition, A.R.-M.;

Funding: This research was funded by Medical University of Lublin DS440/2018-2019 grant, The Polish Ministry of Science and Higher Education MNmb 402/2018-2019, MNmb 510/2016-2017 grants and Polish National Science Centre (NCN): DEC-2015/17/B/NZ1/01777 and DEC2017/25/B/NZ4/02364. Narodowa Agencja Wymiany Akademickiej PolFinGerS 13/2019.

Data Availability Statement: The authors confirm that the data supporting the findings of this study are available within the article. Raw data are included as a Excel file in Supplementary Materials section.

Acknowledgments: We would like to thank Tuula Salo for the myogel.

Conflicts of Interest: The authors declare no conflict of interest.

\section{References}

1. Qin, Q.; Xu, Y.; He, T.; Qin, C.; Xu, J. Normal and disease-related biological functions of Twist1 and underlying molecular mechanisms. Cell Res. 2012, 22, 90-106, doi:10.1038/cr.2011.144.

2. Hirsch, N.; Eshel, R.; Bar Yaacov, R.; Shahar, T.; Shmulevich, F.; Dahan, I.; Levaot, N.; Kaplan, T.; Lupiáñez, D.G.; Birnbaum, R.Y. Unraveling the transcriptional regulation of TWIST1 in limb development. PLoS Genet. 2018, 14, 1-20, doi:10.1371/journal.pgen.1007738.

3. Pelc, A.; Mikulewicz, M. Saethre-chotzen syndrome: Case report and literature review. Dent. Med. Probl. 2018, 55, 217-225, doi:10.17219/dmp/91050.

4. Norozi, F.; Ahmadzadeh, A.; Shahjahani, M.; Shahrabi, S.; Saki, N. Twist as a new prognostic marker in hematological malignancies. Clin. Transl. Oncol. 2016, 18, 113-124, doi:10.1007/s12094-015-1357-0.

5. $\quad$ Alexander, N.R.; Tran, N.L.; Rekapally, H.; Summers, C.E.; Glackin, C.; Heimark, R.L. N-cadherin gene expression in prostate carcinoma is modulated by integrin-dependent nuclear translocation of Twist1. Cancer Res. 2006, 66, 3365-3369, doi:10.1158/0008-5472.CAN-05-3401.

6. Vesuna, F.; van Diest, P.; Chen, J.H.; Raman, V. Twist is a transcriptional repressor of E-cadherin gene expression in breast cancer. Biochem. Biophys. Res. Commun. 2008, 367, 235-241, doi:10.1016/j.bbrc.2007.11.151.

7. $\quad$ Fu, J.; Qin, L.; He, T.; Qin, J.; Hong, J.; Wong, J.; Liao, L.; Xu, J. The TWIST/Mi2/NuRD protein complex and its essential role in cancer metastasis. Cell Res. 2011, 21, 275-289, doi:10.1038/cr.2010.118.

8. Wang, J.; Nikhil, K.; Viccaro, K.; Chang, L.; Jacobsen, M.; Sandusky, G.; Shah, K. The Aurora-A-Twist1 axis promotes highly aggressive phenotypes in pancreatic carcinoma. J. Cell Sci. 2017, 130, 1078-1093, doi:10.1242/jcs.196790.

9. Zhou, J.; Zhang, J.; Xu, M.; Ke, Z.; Zhang, W.; Mai, J. High SRC-1 and Twist1 expression predicts poor prognosis and promotes migration and invasion by inducing epithelial-mesenchymal transition in human nasopharyngeal carcinoma. PLoS One 2019, 14, e0215299, doi:10.1371/journal.pone.0215299.

10. Zhao, Z.; Rahman, M.A.; Chen, Z.G.; Shin, D.M. Multiple biological functions of Twist1 in various cancers. Oncotarget 2017, 8, 20380-20393, doi:10.18632/oncotarget.14608.

11. Piccinin, S.; Tonin, E.; Sessa, S.; Demontis, S.; Rossi, S.; Pecciarini, L.; Zanatta, L.; Pivetta, F.; Grizzo, A.; Sonego, M.; et al. A “Twist box" Code of p53 Inactivation: Twist box:p53 Interaction Promotes p53 Degradation. Cancer Cell 2012, 22, 404-415, doi:10.1016/j.ccr.2012.08.003.

12. Kim, K.; Park, E.Y.; Yoon, M.S.; Suh, D.S.; Kim, K.H.; Lee, J.H.; Shin, D.H.; Kim, J.Y.; Sol, M.Y.; Choi, K.U. The role of TW IST in ovarian epithelial cancers. Korean J. Pathol. 2014, 48, 283-291, doi:10.4132/KoreanJPathol.2014.48.4.283.

13. Kwok, W.K.; Ling, M.T.; Lee, T.W.; Lau, T.C.M.; Zhou, C.; Zhang, X.; Chua, C.W.; Chan, K.W.; Chan, F.L.; Glackin, C.; et al. Up-regulation of TWIST in prostate cancer and its implication as a therapeutic target. Cancer Res. 2005, 65, 5153-5162, 


\section{doi:10.1158/0008-5472.CAN-04-3785.}

14. Yuen, H.F.; Kwok, W.K.; Chan, K.K.; Chua, C.W.; Chan, Y.P.; Chu, Y.Y.; Wong, Y.C.; Wang, X.; Chan, K.W. TWIST modulates prostate cancer cell-mediated bone cell activity and is upregulated by osteogenic induction. Carcinogenesis 2008, 29, 1509_ 1518, doi:10.1093/carcin/bgn105.

15. Chen, P.C.; Tai, H.C.; Lin, T.H.; Wang, S.W.; Lin, C.Y.; Chao, C.C.; Yu, H.J.; Tsai, Y.C.; Lai, Y.W.; Lin, C.W.; et al. CCN3 promotes epithelial-mesenchymal transition in prostate cancer via FAK/Akt/HIF-1 $\alpha$-induced twist expression. Oncotarget 2017, 8, 74506-74518, doi:10.18632/oncotarget.20171.

16. Lyu, P.; Zhang, S.D.; Yuen, H.F.; McCrudden, C.M.; Wen, Q.; Chan, K.W.; Kwok, H.F. Identification of TWIST-interacting genes in prostate cancer. Sci. China Life Sci. 2017, 60, 386-396, doi:10.1007/s11427-016-0262-6.

17. Shibata, K.; Kajiyama, H.; Ino, K.; Terauchi, M.; Yamamoto, E.; Nawa, A.; Nomura, S.; Kikkawa, F. Twist expression in patients with cervical cancer is associated with poor disease outcome. Ann. Oncol. 2008, 19, 81-85, doi:10.1093/annonc/mdm344.

18. Fondrevelle, M.E.; Kantelip, B.; Reiter, R.E.; Chopin, D.K.; Thiery, J.P.; Monnien, F.; Bittard, H.; Wallerand, H. The expression of Twist has an impact on survival in human bladder cancer and is influenced by the smoking status. Urol. Oncol. Semin. Orig. Investig. 2009, 27, 268-276, doi:10.1016/j.urolonc.2007.12.012.

19. Song, Y.H.; Shiota, M.; Yokomizo, A.; Uchiumi, T.; Kiyoshima, K.; Kuroiwa, K.; Oda, Y.; Naito, S. Twist1 and Y-box-binding protein-1 are potential prognostic factors in bladder cancer. Urol. Oncol. Semin. Orig. Investig. 2014, 32, 31.e1-31.e7, doi:10.1016/j.urolonc.2012.11.003.

20. Niu, R.F.; Zhang, L.; Xi, G.M.; Wei, X.Y.; Yang, Y.; Shi, Y.R.; Hao, X.S. Up-regulation of twist induces angiogenesis and correlates with metastasis in hepatocellular carcinoma. J. Exp. Clin. Cancer Res. 2007, 26, 385-394.

21. Abdel Raouf, S.M.; Ibrahim, T.R.; Abdelaziz, L.A.; Farid, M.I.; Mohamed, S.Y. Prognostic Value of TWIST1 and EZH2 Expression in Colon Cancer. J. Gastrointest. Cancer 2019, doi:10.1007/s12029-019-00344-4.

22. Galván, J.A.; Helbling, M.; Koelzer, V.H.; Tschan, M.P.; Berger, M.D.; Hädrich, M.; Schnüriger, B.; Karamitopoulou, E.; Dawson, H.; Inderbitzin, D.; et al. TWIST1 and TWIST2 promoter methylation and protein expression in tumor stroma influence the epithelial-mesenchymal transition-like tumor budding phenotype in colorectal cancer. Oncotarget 2015, 6, 874885, doi:10.18632/oncotarget.2716.

23. Lee, K.W.; Kim, J.H.; Han, S.; Sung, C.O.; Do, I.G.; Ko, Y.H.; Um, S.H.; Kim, S.H. Twist1 is an independent prognostic factor of esophageal squamous cell carcinoma and associated with its epithelial-mesenchymal transition. Ann. Surg. Oncol. 2012, 19, 326-335, doi:10.1245/s10434-011-1867-0.

24. Fahim, Y.; Yousefi, M.; Izadpanah, M.H.; Forghanifard, M.M. TWIST1 correlates with Notch signaling pathway to develop esophageal squamous cell carcinoma. Mol. Cell. Biochem. 2020, 474, 181-188, doi:10.1007/s11010-020-03843-2.

25. Ardalan Khales, S.; Abbaszadegan, M.R.; Majd, A.; Forghanifard, M.M. TWIST1 upregulates matrix metalloproteinase (MMP) genes family in esophageal squamous carcinoma cells. Gene Expr. Patterns 2020, 37, 119127, doi:10.1016/j.gep.2020.119127.

Zhang, L.; Su, B.; Sun, W.; Li, W.; Luo, M.; Liu, D.; Mei, Q.; Long, G.; Hu, G.; Hu, G. Twist1 promotes radioresistance in nasopharyngeal carcinoma. Oncotarget 2016, 7, 81332-81340, doi:10.18632/oncotarget.12875.

Elias, M.C.; Tozer, K.R.; Silber, J.R.; Mikheeva, S.; Deng, M.; Morrison, R.S.; Manning, T.C.; Silbergeld, D.L.; Glackin, C.A.; Reh, T.A.; et al. TWIST is expressed in human gliomas and promotes invasion. Neoplasia 2005, 7, 824-837, doi:10.1593/neo.04352.

28. Nordfors, K.; Haapasalo, J.; Mäkelä, K.; Granberg, K.J.; Nykter, M.; Korja, M.; Paavonen, T.; Haapasalo, H.; Soini, Y. Twist predicts poor outcome of patients with astrocytic glioma. J. Clin. Pathol. 2015, 68, 905-912, doi:10.1136/jclinpath-2015-202868.

29. Wang, N.; Guo, D.; Zhao, Y.Y.; Dong, C.Y.; Liu, X.Y.; Yang, B.X.; Wang, S.W.; Wang, L.; Liu, Q.G.; Ren, Q.; et al. TWIST-1 promotes cell growth, drug resistance and progenitor clonogenic capacities in myeloid leukemia and is a novel poor prognostic factor in acute myeloid leukemia. Oncotarget 2015, 6, 20977-20992, doi:10.18632/oncotarget.4007. 
30. Wushou, A.; Hou, J.; Zhao, Y.-J.; Shao, Z.-M. Twist-1 Up-Regulation in Carcinoma Correlates to Poor Survival. Int. J. Mol. Sci. 2014, 15, 21621-21630, doi:10.3390/ijms151221621.

31. Khan, M.A.; Chen, H.C.; Zhang, D.; Fu, J. Twist: A molecular target in cancer therapeutics. Tumor Biol. 2013, 34, 2497-2506, doi:10.1007/s13277-013-1002-x.

32. Shi, J.; Cao, J.; Zhou, B. Twist-BRD4 Complex: Potential Drug Target for Basal-like Breast Cancer. Curr. Pharm. Des. 2015, 21, 1256-1261, doi:10.2174/1381612821666141211153853.

33. Liu, T.; Zhao, X.; Zheng, X.; Zheng, Y.; Dong, X.; Zhao, N.; Liao, S.; Sun, B. The EMT transcription factor, Twist1, as a novel therapeutic target for pulmonary sarcomatoid carcinomas. Int. J. Oncol. 2020, 56, 750-760, doi:10.3892/ijo.2020.4972.

34. Yuan, T.; Ling, F.; Wang, Y.; Teng, Y. A natural product atalantraflavone inhibits non-small cell lung cancer progression via destabilizing Twist1. Fitoterapia 2019, 137, 104275, doi:10.1016/j.fitote.2019.104275.

35. Wang, S.C.; Sun, H.L.; Hsu, Y.H.; Liu, S.H.; Lii, C.K.; Tsai, C.H.; Liu, K.L.; Huang, C.S.; Li, C.C. $\alpha$-Linolenic acid inhibits the migration of human triple-negative breast cancer cells by attenuating Twist1 expression and suppressing Twist1-mediated epithelial-mesenchymal transition. Biochem. Pharmacol. 2020, 180, 114152, doi:10.1016/j.bcp.2020.114152.

36. Lim, S.-C.; Hwang, H.; Han, S.I. Ellagic Acid Inhibits Extracellular Acidity-Induced Invasiveness and Expression of COX1, COX2, Snail, Twist 1, and c-myc in Gastric Carcinoma Cells. Nutrients 2019, 11, 3023, doi:10.3390/nu11123023.

37. Menéndez-Menéndez, J.; Hermida-Prado, F.; Granda-Díaz, R.; González, A.; García-Pedrero, J.M.; Del-Río-Ibisate, N.; González-González, A.; Cos, S.; Alonso-González, C.; Martínez-Campa, C. Deciphering the Molecular Basis of Melatonin Protective Effects on Breast Cells Treated with Doxorubicin: TWIST1 a Transcription Factor Involved in EMT and Metastasis, a Novel Target of Melatonin. Cancers (Basel). 2019, 11, 1011, doi:10.3390/cancers11071011.

38. Gajula, R.P.; Chettiar, S.T.; Williams, R.D.; Thiyagarajan, S.; Kato, Y.; Aziz, K.; Wang, R.; Gandhi, N.; Wild, A.T.; Vesuna, F.; et al. The Twist box domain is required for Twist1-induced prostate cancer metastasis. Mol. Cancer Res. 2013, 11, 1387-1400, doi:10.1158/1541-7786.MCR-13-0218-T.

39. Ran FA, Hsu PD, Wright J, Agarwala V, Scott DA, Z.F. Genome engineering using the CRISPR-Cas9 system. Nat. Protoc. 2006, 8, 1-10, doi:10.1038/nprot.2013.143.Genome.

40. Sun, Y.; Vandenbriele, C.; Kauskot, A.; Verhamme, P.; Hoylaerts, M.F.; Wright, G.J. A Human Platelet Receptor Protein Microarray Identifies the High Affinity Immunoglobulin E Receptor Subunit $\alpha(\mathrm{FccR} 1 \alpha)$ as an Activating Platelet Endothelium Aggregation Receptor 1 (PEAR1) Ligand. Mol. Cell. Proteomics 2015, 14, 1265-1274, doi:10.1074/mcp.M114.046946.

41. Miranda, K.C.; Khromykh, T.; Christy, P.; Le, T.L.; Gottardi, C.J.; Yap, A.S.; Stow, J.L.; Teasdale, R.D. A Dileucine Motif Targets E-cadherin to the Basolateral Cell Surface in Madin-Darby Canine Kidney and LLC-PK1 Epithelial Cells. J. Biol. Chem. 2001, 276, 22565-22572, doi:10.1074/jbc.M101907200.

42. Zhou, B.P.; Deng, J.; Xia, W.; Xu, J.; Li, Y.M.; Gunduz, M.; Hung, M.C. Dual regulation of Snail by GSK-3 $\beta$-mediated phosphorylation in control of epithelial-mesenchymal transition. Nat. Cell Biol. 2004, 6, 931-940, doi:10.1038/ncb1173.

43. Trehan, A.; Kiełbus, M.; Czapinski, J.; Stepulak, A.; Huhtaniemi, I.; Rivero-Müller, A. REPLACR-mutagenesis, a one-step method for site-directed mutagenesis by recombineering. Sci. Rep. 2016, 6, doi:10.1038/srep19121.

44. Kiełbus, M.; Czapiński, J.; Kałafut, J.; Woś, J.; Stepulak, A.; Rivero-Müller, A. Genetically Engineered Lung Cancer Cells for Analyzing Epithelial-Mesenchymal Transition. Cells 2019, 8, 1-17, doi:10.3390/cells8121644.

45. Salo, T.; Sutinen, M.; Hoque Apu, E.; Sundquist, E.; Cervigne, N.K.; de Oliveira, C.E.; Akram, S.U.; Ohlmeier, S.; Suomi, F.; Eklund, L.; et al. A novel human leiomyoma tissue derived matrix for cell culture studies. BMC Cancer 2015, 15, 1, doi:10.1186/s12885-015-1944-z.

46. Naakka, E.; Tuomainen, K.; Wistrand, H.; Palkama, M.; Suleymanova, I.; Al-Samadi, A.; Salo, T. Fully human tumor-based matrix in three-dimensional spheroid invasion assay. J. Vis. Exp. 2019, 2019, doi:10.3791/59567.

47. Portales-Casamar, E.; Arenillas, D.; Lim, J.; Swanson, M.I.; Jiang, S.; McCallum, A.; Kirov, S.; Wasserman, W.W. The PAZAR 
database of gene regulatory information coupled to the ORCA toolkit for the study of regulatory sequences. Nucleic Acids Res. 2009, 37, 54-60, doi:10.1093/nar/gkn783.

48. Tannous, B.A.; Kim, D.E.; Fernandez, J.L.; Weissleder, R.; Breakefield, X.O. Codon-optimized gaussia luciferase cDNA for mammalian gene expression in culture and in vivo. Mol. Ther. 2005, 11, 435-443, doi:10.1016/j.ymthe.2004.10.016.

49. Zhao, Z.; Rahman, M.A.; Chen, Z.G.; Shin, D.M. Multiple biological functions of Twist1 in various cancers. Oncotarget 2017, 8, 20380-20393, doi:10.18632/oncotarget.14608.

50. Palumbo-Zerr, K.; Soare, A.; Zerr, P.; Liebl, A.; Mancuso, R.; Tomcik, M.; Sumova, B.; Dees, C.; Chen, C.W.; Wohlfahrt, T.; et al. Composition of TWIST1 dimers regulates fibroblast activation and tissue fibrosis. Ann. Rheum. Dis. 2017, 76, 244-251, doi:10.1136/annrheumdis-2015-208470.

51. Hsu, K.W.; Hsieh, R.H.; Huang, K.H.; Li, A.F.Y.; Chi, C.W.; Wang, T.Y.; Tseng, M.J.; Wu, K.J.; Yeh, T.S. Activation of the Notch1/STAT3/Twist signaling axis promotes gastric cancer progression. Carcinogenesis 2012, 33, 1459-1467, doi:10.1093/carcin/bgs165.

52. Xu, R.; Won, J.Y.; Kim, C.H.; Kim, D.E.; Yim, H. Roles of the Phosphorylation of Transcriptional Factors in EpithelialMesenchymal Transition. J. Oncol. 2019, 2019, doi:10.1155/2019/5810465.

53. Franco, H.L.; Casasnovas, J.; Rodríguez-Medina, J.R.; Cadilla, C.L. Redundant or separate entities? - Roles of Twist1 and Twist2 as molecular switches during gene transcription. Nucleic Acids Res. 2011, 39, 1177-1186, doi:10.1093/nar/gkq890.

54. Riemann, A.; Rauschner, M.; Gießelmann, M.; Reime, S.; Haupt, V.; Thews, O. Extracellular Acidosis Modulates the Expression of Epithelial-Mesenchymal Transition (EMT) Markers and Adhesion of Epithelial and Tumor Cells. Neoplasia (United States) 2019, 21, 450-458, doi:10.1016/j.neo.2019.03.004.

55. Hu, J.; Tian, J.; Zhu, S.; Sun, L.; Yu, J.; Tian, H.; Dong, Q.; Luo, Q.; Jiang, N.; Niu, Y.; et al. Sox5 contributes to prostate cancer metastasis and is a master regulator of TGF- $\beta$-induced epithelial mesenchymal transition through controlling Twist1 expression. Br. J. Cancer 2018, 118, 88-97, doi:10.1038/bjc.2017.372.

56. Shao, T.; Song, P.; Hua, H.; Zhang, H.; Sun, X.; Kong, Q.; Wang, J.; Luo, T.; Jiang, Y. Gamma synuclein is a novel Twist1 target that promotes TGF- $\beta$-induced cancer cell migration and invasion article. Cell Death Dis. 2018, 9, 1-13, doi:10.1038/s41419-0180657-z.

57. Park, M.J.; Lee, D.E.; Shim, M.K.; Jang, E.H.; Lee, J.K.; Jeong, S.Y.; Kim, J.H. Piperlongumine inhibits TGF- $\beta$-induced epithelial-to-mesenchymal transition by modulating the expression of E-cadherin, Snail1, and Twist1. Eur. J. Pharmacol. 2017, 812, 243-249, doi:10.1016/j.ejphar.2017.07.036.

58. Zheng, H.; Fu, Y.; Yang, T. Propofol inhibits proliferation, migration, and invasion of hepatocellular carcinoma cells by downregulating Twist. J. Cell. Biochem. 2019, 120, 12803-12809, doi:10.1002/jcb.28551.

59. Ma, G.; Ma, G.; He, J.; Yu, Y.; Xu, Y.; Xu, Y.; Yu, X.; Martinez, J.; Lonard, D.M.; Xu, J.; et al. Tamoxifen inhibits ER-negative breast cancer cell invasion and metastasis by accelerating twist1 degradation. Int. J. Biol. Sci. 2015, 11, 618-628, doi:10.7150/ijbs.11380.

60. Mammoto, T.; Jiang, A.; Jiang, E.; Mammoto, A. Role of Twist1 phosphorylation in angiogenesis and pulmonary fibrosis. Am. J. Respir. Cell Mol. Biol. 2016, 55, 633-644, doi:10.1165/rcmb.2016-0012OC.

61. Li, J.; Liu, C.H.; Sun, Y.; Gong, Y.; Fu, Z.; Evans, L.P.; Tian, K.T.; Juan, A.M.; Hurst, C.G.; Mammoto, A.; et al. Endothelial TWIST1 promotes pathological ocular angiogenesis. Investig. Ophthalmol. Vis. Sci. 2014, 55, 8267-8277, doi:10.1167/iovs.1415623.

62. Wang, Z.; Li, J.; Li, K.; Xu, J. SOX6 is downregulated in osteosarcoma and suppresses the migration, invasion and epithelialmesenchymal transition via TWIST1 regulation. Mol. Med. Rep. 2018, 17, 6803-6811, doi:10.3892/mmr.2018.8681.

63. Liang, Y.J.; Sun, C.P.; Hsu, Y.C.; Chen, Y.W.; Wang, I.A.; Su, C.W.; Tao, M.H.; Wu, J.C. Statin inhibits large hepatitis delta antigen-Smad3-twist-mediated epithelial-to-mesenchymal transition and hepatitis D virus secretion. J. Biomed. Sci. 2020, 27, doi:10.1186/s12929-020-00659-6. 
64. Lo, H.W.; Hsu, S.C.; Xia, W.; Cao, X.; Shih, J.Y.; Wei, Y.; Abbruzzese, J.L.; Hortobagyi, G.N.; Hung, M.C. Epidermal growth factor receptor cooperates with signal transducer and activator of transcription 3 to induce epithelial-mesenchymal transition in cancer cells via up-regulation of TWIST gene expression. Cancer Res. 2007, 67, 9066-9076, doi:10.1158/0008-5472.CAN-070575 .

65. Yu, H.; Gao, G.; Cai, J.; Song, H.; Ma, Z.; Jin, X.; Ji, W.; Pan, B. MiR-539 functions as a tumor suppressor in pancreatic cancer by targeting TWIST1. Exp. Mol. Pathol. 2019, 108, 143-149, doi:10.1016/j.yexmp.2019.04.012.

66. Xiong, Y.; Chen, R.; Wang, L.; Wang, S.; Tu, Y.; Zhu, L.; Wang, C. Downregulation of miR-186 promotes the proliferation and drug resistance of glioblastoma cells by targeting Twist1. Mol. Med. Rep. 2019, 19, 5301-5308, doi:10.3892/mmr.2019.10207.

67. Tuomainen, K.; Al-Samadi, A.; Potdar, S.; Turunen, L.; Turunen, M.; Karhemo, P.-R.; Bergman, P.; Risteli, M.; Åström, P.; Tiikkaja, R.; et al. Human Tumor-Derived Matrix Improves the Predictability of Head and Neck Cancer Drug Testing. Cancers (Basel). 2019, 12, doi:10.3390/cancers12010092.

68. Osorio, L.A.; Farfán, N.M.; Castellón, E.A.; Contreras, H.R. SNAIL transcription factor increases the motility and invasive capacity of prostate cancer cells. Mol. Med. Rep. 2016, 13, 778-786, doi:10.3892/mmr.2015.4585. 